\title{
Hydroelasticity Analysis in Frequency Domain and Time Domain
}

Frank Lin

Lloyd's Register Applied Technology Group, Halifax B3J3J8, Canada

\begin{abstract}
Hydroelasticity has been introduced in ship seakeeping assessment for more than three decades, and it finally becomes an essential tool in marine industry for design of some types of ship. In the 35 years of evolution, hydroelasticity methods applied in industry of marine and offshore energy grown up from two dimensional to three dimensional and now has analysis models of linear model in frequency domain and nonlinear model in time domain. In this paper, we present the three dimensional hydroelasticity theory model in frequency domain and time domain, show the difference in the approach, and discuss their applications in wave-structure interaction.
\end{abstract}

Key words: Hydroelasticity, springing, frequency domain, time domain, boundary element method, linear, nonlinear.

\section{Introduction}

In a design process of floating structures, like ships and offshore structures, hydrodynamic analysis of wave-structure interaction is the first important key step. Method of rigid-body based seakeeping analysis is applied successfully in this type of work for many decades, but suffering failures on some of latest mega-ships, like a container ship over 350 meters in length. It has been found that the computed fatigue life of a large container ship based on a rigid-body hydrodynamic approach is significantly longer than the observation. The elastic-body based analysis method is raised to the stage for the interaction of water waves and elastic structures. An ultimate hydroelasticity solution comes from CFD approach, but it is too expensive to be applied in routine work, because the number of required regular wave cases will be in general 3,000 to 5,000 in a design process, the number of combination of ship speed, wave headings and sea states will be a few hundreds and leads to simulation over hundreds of thousands of hours of real time. The boundary element type of hydroelasticity models remain as the only tool for

Corresponding author: Frank Lin, technical specialist, research field: marine hydrodynamics. routine work. In this paper, general approach of 3D hydroelasticity is presented. Differences between the rigid-body approach and hydroelasticity approach are discussed. We also look into theoretical details of frequency domain hydroelasticity model orientated for conditions of low and moderate sea state, and that of time domain hydroelasticity model orientated for high sea state conditions.

\section{Methodology of Hydroelasticity}

Reliable prediction of hydrodynamic structure load is the key of a successful strength assessment for a structure operated in waves. The hydrodynamic pressure is determined by the location and velocity of wetted surface of the stricture. Rigid-body approach of the structure will be accurate enough if the elastic deformation of the structure wetted surface is small comparing to that induced by rigid-body motion. Elastic deformation needs to be considered in the boundary condition of boundary value problem of flow solution for ships or structures with less stiffness, such as a container ship longer than 350 meters. Hydroelasticity method has been developed for the interaction of waves and elastic structure. Due to the interaction for the flow and structure motion and 
deformation, the hydrodynamic problem and structure dynamic problem is coupled together and needs to be solved at the same time. Direct finite element structure analysis model can be combined with flow solver like RANS or BEM in time domain to form a robust nonlinear tool for hydroelastic assessment, but it will be too expensive to be applied for routine work of a vessel design. FEM based modal analysis is usually used for the structure assessment part, and its solution, eigen-values and eigen vectors are used with boundary element model of hydrodynamic analysis, and forms the so-called hydroelasticity model in seakeeping area. The first two dimensional frequency domain hydroelastic method was proposed by Bishop and Price in 1979 [1]. In their method, a ship was represented by a Timoshenko beam and discretized to a number of 2D beam elements for structure analysis part, and the wave flow solution around the ship was determined by strip theory. Bishop and Price's work was further developed to 3D by Wu [2], and extended to time domain by many researchers like Wang [3]. The hydroelasticity method has been keeping developing to today, with 3D full structure FEM model with 3D BEM model for hydrodynamic solution in both frequency domain and time domain.

In a finite element model of a structure, stress in an element can be estimated by the displacement of node points of the element. Vector of model node displacement, $\mathbf{U}$, can be determined from the model elastic motion equation

$$
[\mathbf{M}]\{\ddot{\mathbf{U}}\}+[\mathbf{B}]\{\dot{\mathbf{U}}\}+[\mathbf{K}]\{\mathbf{U}\}=\{\mathbf{P}\}+\{\mathbf{F}\}+\{\mathbf{G}\}
$$

where $[\mathbf{M}],[\mathbf{B}]$ and $[\mathbf{K}]$ are the matrix of model mass, structural damping and stiffness; $\{\mathbf{P}\}$ is the vector of external surface force; $\{\mathbf{F}\}$ is the vector of external concentrate force, and $\{\mathbf{G}\}$ the vector of external mass force. Dot presents the gradient w.r.t time.

Introducing the homogenous solution of node displacement vector, $\{\mathbf{U}\}=\{\mathbf{D}\} e^{-i \omega t}$ and ignoring structural damping, and all external forcing terms from Eq. (1), solution of equation

$$
\left(-\omega^{2}[\mathbf{M}]+[\mathbf{K}]\right)\{\mathbf{D}\}=\{\mathbf{0}\}
$$

gives the eigen value $\omega_{r}$ and eigen vector $\{\mathbf{D}\}_{r}$ that define dry eigen modes. The number of eigen modes of a FEA model will be the same to the number of model freedoms, six times of the number of node points. Displacement at point $(x, y, z)$ can be expressed by those dry eigen modes in terms of summary

$$
\{\mathbf{U}(x, y, z ; t)\}=[\mathbf{D}(x, y, z)]\{\mathbf{q}(t)\}=\sum_{r} \mathbf{D}_{r}(x, y, z) q_{r}(t),
$$

$q_{r}(t)$ is the amplitude of mode $r$, so-called general coordinate, and

$$
\{\mathbf{D}\}_{r}^{j}=\left(\mathbf{u}^{r}, \vec{\vartheta}^{r}\right)_{j}^{T}=\left(u_{r}, v_{r}, w_{r}, \alpha_{r}, \beta_{r}, \chi_{r}\right)_{j}^{T}
$$

is the displacement of point $j$ induced by mode $r$ with unit modal amplitude.

Multiplying $[\mathbf{D}]^{T}$ on each terms of Eq. (1), and right multiplying [D] on matrix of model mass, damping and stiffness, we have an equation for determine the modal amplitude

$$
[\mathbf{m}]\{\ddot{\mathbf{q}}\}+[\mathbf{b}]\{\dot{\mathbf{q}}\}+[\mathbf{k}]\{\mathbf{q}\}=\{\mathbf{p}\}+\{\mathbf{f}\}+\{\mathbf{g}\},
$$

One of advantages of using dry eigen mode approach is the modal orthogonally. For any elastic dry modes $r$ and $s$, using Kronecker delta, we have

$$
\begin{aligned}
\{\mathbf{D}\}_{r}^{T}[\mathbf{M}]\{\mathbf{D}\}_{s} & =\delta_{r s} m_{r s} \\
\{\mathbf{D}\}_{r}^{T}[\mathbf{K}]\{\mathbf{D}\}_{s} & =\delta_{r s} k_{r s}
\end{aligned}
$$

$m_{r r}$ and $k_{r r}$ is the modal mass and modal stiffness.

We can solve the modal amplitude by applying a location and velocity given by Eq. (3) on wetted surface in a hydrodynamic analysis model and expressing and estimating the three forcing terms in the hydrodynamic model.

In a boundary element hydrodynamic model, linearized boundary surface condition for unsteady velocity potential $\Phi^{U}$ can be given by the surface displacement $(\mathbf{u}, \vec{\vartheta})$ and the steady flow velocity $\mathbf{W}$

$$
\frac{\partial \Phi^{U}}{\partial n}=\left[\frac{\partial \mathbf{u}}{\partial t}+\vec{\vartheta} \times \mathbf{W}-(\mathbf{u} \bullet \nabla) \mathbf{W}\right] \bullet \mathbf{n}+O\left(|\mathbf{u}|^{2}\right),
$$


here $\mathbf{n}$ is the surface normal vector, and surface displacement $(\mathbf{u}, \vec{\vartheta})$ can be estimated from the modal shape functions, or eigen vectors, of the model.

Another fact in a hydroelastic model worth to note is that the eigen mode with nonzero displacement on wetted surface will receive hydrodynamic pressure force, and we call these modes the "wettable mode". All other modes, "non-wettable modes", have no external force from hydrodynamic pressure. The wettable modes will be coupled to each other through hydrodynamic pressure force. Which means the motion of $i$-th wettable mode will induce surface forcing term on $j$-th wettable mode. On the other hand, non-wettable modes are uncoupled. In a hydroelastic model, we only need to consider those wettable modes, usually only the first few wettable modes in a practice.

Another difference between a rigid structure and elastic structure is that the center of gravity of the structure is a point fixed with the structure any more for the elastic case. This change leads to a much complicated equation for the rigid body motion mode.

Let's introduce two Cartesian coordinate systems: the body-fixed frame, HMF, $\tilde{o}-\tilde{x} \tilde{y} \tilde{z}$ with $\tilde{x}$-axis points to the bow, $\tilde{o}-\tilde{x} \tilde{y}$ coordinate plane lying on the undisturbed water surface when the ship has no oscillations, and $\tilde{z}$-axis is positive upward; the last one, the moving reference frame, HRF, $o-x y z$, which is an inertial frame moving at the constant ship speed $U$ and will be identical with the body-fixed frame if the ship has no oscillations. The coordinates of the body-fixed frame origin, $\widetilde{o}$, in the reference frame HMF, namely $\vec{\eta}=\left(\eta_{1}, \eta_{2}, \eta_{3}\right)$ define the translational motion of the ship, so called Surge, Sway and Heave. Three Euler angles $\left(\eta_{4}, \eta_{5}, \eta_{6}\right)$ between the body-fixed frame $\tilde{o}-\tilde{x} \tilde{y} \tilde{z}$ and the reference frame $o-x y z$ define the rotational motion of the ship, so called Roll, Pitch and Yaw. Suppose $\tilde{o}-\tilde{x} \tilde{y} \tilde{z}$ rotates from the position of $o-x y z$ with the angle $\eta_{6}$ about $\tilde{z}$-axis first, then the angle $\eta_{5}$ about $\tilde{y}$-axis, and finally the angle $\eta_{4}$ about $\tilde{x}$-axis, we will have the relation between $\tilde{o}-\tilde{x} \tilde{y} \tilde{z}$ and $o-x y z$ as follow

$$
\left(\begin{array}{l}
x \\
y \\
z
\end{array}\right)=\left(\begin{array}{l}
\eta_{1} \\
\eta_{2} \\
\eta_{3}
\end{array}\right)+\mathbf{R}\left(\begin{array}{c}
\tilde{x} \\
\tilde{y} \\
\tilde{z}
\end{array}\right)
$$

where $\mathbf{R}$ is the mapping matrix defined by the three Euler angles.

By setting the origin on the gravitational center of the structure with zero elastic formation, the equations for rigid body motion, translational and rotational, can be given by

$$
\begin{gathered}
M \frac{d^{2} \vec{\eta}}{d t^{2}}+\mathbf{R} \sum_{s} \frac{d^{2} \eta_{s}}{d t^{2}} \mathbf{M}_{s}+\mathbf{R}\left\{\frac{d \mathbf{w}}{d t} \times\left(\sum_{s} \eta_{s} \mathbf{M}_{s}\right)\right. \\
\left.+\mathbf{w} \times\left[\mathbf{w} \times\left(\sum_{s} \eta_{s} \mathbf{M}_{s}\right)\right]+2 \mathbf{w} \times\left(\sum_{s} \frac{d \eta_{s}}{d t} \mathbf{M}_{s}\right)\right\}=\mathbf{F} \\
M\left\{\left(\sum_{s} \eta_{s} \tilde{\mathbf{r}}_{s}^{g}\right) \times \frac{d^{2}}{d t^{2}}+\left(\sum_{s} \frac{d \eta_{s}}{d t} \tilde{\mathbf{r}}_{s}^{g}\right) \times \frac{d}{d t}\right. \\
\left.+\mathbf{w} \times\left[\left(\sum_{s} \eta_{s} \tilde{\mathbf{r}}_{s}^{g}\right) \times \frac{d}{d t}\right]\right\}\left[\mathbf{R}^{T}(\vec{\eta})\right] \\
+\mathbf{J}\left(\frac{d \mathbf{w}}{d t}\right)+\mathbf{w} \times \mathbf{J}(\mathbf{w})+\sum_{s} \frac{d^{2} \eta_{s}}{d t^{2}}\left[\mathbf{J}_{s}+\mathbf{I}_{s}\left(\vec{\vartheta}^{s}\right)\right]=\mathbf{M}
\end{gathered}
$$

where $\mathbf{M}$ and $\mathbf{J}$ is the total mass and moment of mass inertial of the structure; $\eta_{s}$ the amplitude of the $s$-th elastic mode; $\mathbf{w}$ the vector of rotation velocity; $\widetilde{\mathbf{r}}_{\mathrm{s}}{ }$, $\mathbf{M}_{s}$, and $\mathbf{J}_{s}$ are the modal mass center, modal "modal mass vector" and "modal mass moment" of the $s$-th elastic mode defined by

$$
\begin{aligned}
& \tilde{\mathbf{r}}_{s}^{g}=\frac{1}{M} \mathbf{M}_{s} ; \quad \mathbf{M}_{s}=\int\left\{\begin{array}{c}
\tilde{u}^{s} \\
\tilde{v}^{s} \\
\tilde{w}^{s}
\end{array}\right\} d m ; \\
& \mathbf{J}_{s}=\int_{M}^{\tilde{\mathbf{r}} \times \tilde{\mathbf{u}}^{s} d m .}
\end{aligned}
$$

“modal moment of mass inertial”, $\mathbf{I}_{s}\left(\vec{\vartheta}^{s}\right)$, represents the effect due to the elastic rotation deformation of the $s$-th elastic mode, which is defined as

$$
\left(\begin{array}{ccc}
\int_{M} \alpha_{s}\left(\tilde{y}^{2}+\tilde{z}^{2}\right) d m & -\int_{M} \beta_{s} \tilde{x} \tilde{y} d m & -\int_{M} \chi_{s} \tilde{x} \tilde{z} d m \\
-\int_{M} \alpha_{s} \tilde{y} \tilde{x} d m & \int_{M} \beta_{s}\left(\tilde{z}^{2}+\tilde{x}^{2}\right) d m & -\int_{M} \chi_{s} \tilde{y} \tilde{z} d m \\
-\int_{M} \alpha_{s} \tilde{z} \tilde{x} d m & -\int_{M} \beta_{s} \tilde{z} \tilde{y} d m & \left.\int_{M} \chi_{s}^{(} \tilde{x}^{2}+\tilde{y}^{2}\right) d m
\end{array}\right) .
$$




\section{Boundary Element Hydroelastic Method in Frequency Domain}

In a linearized frequency domain model, the external disturbance, the wave, is assumed "small" and responses induced by this small disturbance following the time function $e^{-i \omega_{e} t}$, where the encounter frequency $\omega_{e}$ is a function of incident wave frequency $\omega$, ship speed $U$, and wave heading $\chi$

$$
\omega_{e}=\omega-U k \cos \chi,
$$

here wave number $k=\omega^{2} / g, g$ the gravitational acceleration, for deep water, and $\chi=0^{\circ}$ represents the following sea, moving ship and propagating wave has the same direction, and $\chi=180^{\circ}$ represents the head sea condition. Unsteady flow velocity potential is defined by

$$
\begin{array}{r}
\Phi^{U}(\mathbf{r} ; t)=\operatorname{Re}\left\{a e ^ { - i \omega _ { e } t } \left[-i \frac{g}{\omega}\left(\phi_{I}(\mathbf{r})+\phi_{D}(\mathbf{r})\right)\right.\right. \\
\left.\left.-i \omega_{e} \sum_{j=1}^{L} \frac{\xi_{j}}{a} \phi_{j}(\mathbf{r})\right]\right\}
\end{array}
$$

$L$ is the number of system freedom, which equals to 6 for a rigid-body model, and 6 plus the number of involved structural elastic eigen modes in a hydroelasticity model. $\eta_{j}(t)=\operatorname{Re}\left(\xi_{j} e^{-i \omega_{e} t}\right)$ is the displacement of mode $j$ at time $t$, and $\xi_{j}$ is the complex mode amplitude contains information of amplitude and phase. $a$ is the amplitude of incident wave and its space velocity potential for deep water is given by

$$
\phi_{I}(x, y, z)=e^{k z} \exp \{i k[(\cos \chi) x+(\sin \chi) y]\}
$$

The first responsibility of hydroelastic model is determination of the diffraction potential $\phi_{D}(\mathbf{r})$ and radiation potential $\phi_{j}(\mathbf{r})$ for each system freedom. Both diffraction and radiation potential satisfies Laplace equation $\nabla^{2} \phi(\mathbf{r})=0$; linearized free surface condition

$$
\begin{gathered}
{\left[g \frac{\partial}{\partial n}+\left(\frac{\partial}{\partial t}+\mathbf{W} \bullet \nabla\right)^{2}\right] \phi=0,} \\
\text { on clam water surface } z=0,
\end{gathered}
$$

and the radiation condition requires the diffraction and radiation wave due to the existence of the ship propagates outward. The velocity potential in the fluid domain and on the boundary surface can be estimated by an integration of a singularity distribution on wetted hull surface $S_{h}$

$$
\left\{\begin{array}{c}
1 \\
\nabla_{p}
\end{array}\right\} \phi\left(\mathbf{r}_{p}\right)=\iint_{S_{h}} \sigma\left(\mathbf{r}_{q}\right)\left\{\begin{array}{c}
1 \\
\nabla_{p}
\end{array}\right\} G\left(\mathbf{r}_{p}, \mathbf{r}_{q}\right) d s_{q}
$$

$G\left(\mathbf{r}_{p}, \mathbf{r}_{q}\right)$ is the Green's function that satisfies Laplace equation, free surface condition and radiation condition. The strength of singularity $\sigma$ can be solved from boundary integral equation

$$
\frac{\partial \phi\left(\mathbf{r}_{p}\right)}{\partial n_{p}}=\alpha\left(\mathbf{r}_{p}\right) \sigma\left(\mathbf{r}_{p}\right)+\iint_{S_{h}} \sigma\left(\mathbf{r}_{q}\right) \frac{\partial G\left(\mathbf{r}_{p}, \mathbf{r}_{q}\right)}{\partial n_{p}} d s_{q} .
$$

And $\alpha\left(\mathbf{r}_{p}\right)$ is the interior solid angle of field point $\mathbf{r}_{p}$ on wetted hull surface $S_{h}$. The required surface condition for diffraction problem is

$$
\frac{\partial \phi_{D}}{\partial n}=-\frac{\partial \phi_{I}}{\partial n}
$$

and for radiation problem of $j$-th motion/elastic mode

$$
\frac{\partial \phi_{j}}{\partial n}=n_{j}+\frac{1}{i \omega_{e}} m_{j}
$$

Modal normal component $n_{j}$, the so-called n-term, and m-term can be estimated by

$$
\left.\begin{array}{l}
n_{j}=\mathbf{u}^{j} \bullet \mathbf{n} \\
m_{j}=\left[\vec{\vartheta}^{j} \times \mathbf{W}-\left(\mathbf{u}^{j} \bullet \nabla\right) \mathbf{W}\right] \bullet \mathbf{n}
\end{array}\right\} .
$$

Hydrodynamic pressure on wetted hull surface comes from Bernoulli's equation using velocity potential and its gradient. Its linearized form is of

$$
p(\mathbf{r} ; t)-p_{0}=-\rho\left(\frac{\partial \Phi^{U}}{\partial t}+\mathbf{W} \bullet \nabla \Phi^{U}\right)
$$

The hydrodynamic force on mode $i$ can be computed by integration

$$
p_{i}=-\iint_{S_{h}} n_{i}\left[p(\mathbf{r} ; t)-p_{0}\right] d s
$$

After an order analysis of the perturbation expansion of this theoretical approach, this surface modal force can be expressed in terms of modal 
amplitude

$$
\begin{gathered}
p_{i}= \\
\operatorname{Re}\left\{e^{-i \omega_{e} t}\left[E_{i}-\left(i \omega_{e}\right)^{2} \sum_{j=1}^{L} \xi_{j}\left(A_{i j}+\frac{1}{i \omega_{e}} B_{i j}-\frac{1}{\omega_{e}^{2}} C_{i j}\right)\right]\right\}
\end{gathered}
$$

And modal wave exciting force $E_{i}$, modal wave making added-mass and damping coefficient $A_{i j}$ and $B_{i j}$ are computed as follows

$$
\begin{aligned}
E_{i}=\rho g a \iint_{S_{h}} n_{i}\left[\frac{\omega_{e}}{\omega}\left(\phi_{I}+\phi_{D}\right)\right. & \\
& \left.+\frac{1}{i \omega} \mathbf{W} \bullet \nabla\left(\phi_{I}+\phi_{D}\right)\right] d s \\
A_{i j}+\frac{B_{i j}}{i \omega_{e}}=\rho \iint_{S_{h}} n_{i} & {\left[\phi_{j}+\frac{1}{i \omega_{e}} \mathbf{W} \bullet \nabla \phi_{j}\right] d s }
\end{aligned}
$$

Modal restoring coefficient $C_{i j}$ has similar integration formula but much lengthy.

The amplitude of rigid-body motion and elastic mode for a model without concentrate force can be solved then from

$$
\begin{gathered}
\sum_{j=1}^{L}\left[\left(i \omega_{e}\right)^{2}\left(m_{i j}+A_{i j}\right)+i \omega_{e}\left(b_{i j}+B_{i j}+B_{i j}^{V}\right)\right. \\
\left.+\left(k_{i j}+C_{i j}\right)\right] \xi_{j}=E_{i}
\end{gathered}
$$

The rigid-body motion in fact comes from the linearized equation

$$
\begin{aligned}
& M \frac{d^{2} \vec{\eta}}{d t^{2}}+\sum_{s} \frac{d^{2} \eta_{s}}{d t^{2}} \mathbf{M}_{s}=\mathbf{F} \\
& \mathbf{J}\left(\frac{d \mathbf{w}}{d t}\right)+\sum_{s} \frac{d^{2} \eta_{s}}{d t^{2}}\left[\mathbf{J}_{s}+\mathbf{I}_{s}\left(\vec{\vartheta}^{s}\right)\right]=\mathbf{M}
\end{aligned}
$$

Eq. (28) is expressed in a hydrodynamic reference coordinate system. This system is on the clam water surface and moving at the constant speed $U$ toward the ship moving direction. Therefore it is an inertial coordinate system and the mass modal force is vanished, as the existing mass force is gravity and it is a constant in time in this coordinate system. In this deformation/motion equation, $B_{i j}^{V}$ is the coefficient of viscous flow induced damping which is important to those modes having small wave making damping, such as roll motion mode. It is possible to involve nonlinear viscous flow damping in the analysis. $b_{i j}$ is the coefficient of structural modal damping, and it is still a challenge for structure scientists and engineers on how to estimate this damping reliably. So far, it mainly depends on data of model test and sea trail.

In most of available tools, the structure analysis is performed in a ship fixed coordinate system that leads to nonzero mass modal force with two components, one is induced by the inertial acceleration of rigid body ship motion, and the other comes from the dynamic gravitational acceleration in this ship fixed coordinate system. This mass modal force will excite every non-wettable mode and those modes may need to be involved in structure assessment.

\section{Boundary Element Hydroelastic Method in Time Domain}

As described in previous section, frequency domain hydroelastic BEM model combines linear structure FEA, linear hydrodynamic BEM with linear or nonlinear deformation/motion equation. It is basically a linear Hydroelasticity approach. This frequency domain approach works well for a ship or offshore structure in low and moderate sea conditions, but its results become unreliable for large wave case and nonlinear model is required. Note that the dominant factors in hydrodynamic problems of ship or offshore structure in large wave are the vertical shape change of the hull surface, i.e. the hull flare, and high wave itself, a cheaper and more efficient time domain hydroelastic BEM model could be considered in steady of the ultimate CFD model. This time domain BEM model simulates responses in wave by using retardation function, the response function to an impulse disturbance and carries the memory effects of disturbance in past. The retardation function in this time domain BEM model can be determined either by time domain Green's function or by the Fourier transformation of hydrodynamic results obtained from frequency domain analysis. Comparing to the first approach, the second one usually takes shorter 
computing time and requiring much smaller computer memory and it is the method we will use in the work of this paper. The important difference between the frequency domain hydroelastic BEM model and the time domain model is that the small wave restriction is removed in the time domain model, and it leads to large responses like structure motion/deformation, internal load and so on. The nonlinear terms needs to be involved in computations of motion/deformation equation and internal load.

One of the main differences from the linearized frequency domain model is that the rigid-body motion mode can have large amplitude, and Eqs. (9) and (10) are adopted. Also in the time domain model, modal surface force, i.e. the hydrodynamic force, will be determined by

$$
\begin{array}{r}
F_{i}=F_{i}^{r a d}+F_{i}^{d i f}+F_{i}^{F K}+F_{i}^{r s t}+F_{i}^{o t h e r s}, \\
i=1,2, \ldots, L
\end{array}
$$

where subscript $i$ stands for deformation/motion mode; $F_{i}^{\text {rad }}$ is the radiation force on mode $i$; $F_{i}^{\text {dif }}$ is the diffraction force; $F_{i}^{F K}$ is so-called Froude-Krylov force due to incident waves; $F_{i}^{\text {rst }}$ is the restoring force due to hydrostatic pressure; and $F_{i}^{\text {others }}$ represents the force due to all other external effects like mooring, viscous damping, tank sloshing, maneuvering. Different from the frequency domain model, the maneuvering force on rigid-body motion mode for a ship with forward speed is a mandatory factor in the time domain simulation. Similarly the mooring force or position stationary force for a floating offshore platform is also important.

The radiation force on mode $i$ that is induced by the motion/deformation of the structure is determined by the convolution

$$
\begin{gathered}
F_{i}^{r a d}(t)=-\sum_{j=1}^{L}\left[A_{i j}^{\infty} \ddot{\eta}_{j}(t)+B_{i j}^{\infty} \dot{\eta}_{j}(t)+C_{i j}^{\infty} \eta_{j}(t)\right. \\
\left.+\int_{0}^{t} \kappa_{i j}(t-\tau) \dot{\eta}_{j}(\tau) d \tau\right] \\
i=1,2, \ldots, L .
\end{gathered}
$$

$\eta_{j}(t)$ is the displacement of mode $j$ at time $t$,
$A_{i j}^{\infty}, B_{i j}^{\infty}$ and $C_{i j}^{\infty}$ are the coefficient of wave making added-mass, damping and restoring force at infinite encounter frequency. $\kappa_{i j}(t)$ is the retardation function and can be estimated from

$$
\kappa_{i j}(t)=\left\{\begin{array}{l}
\frac{2}{\pi} \int_{0}^{\infty}\left[A_{i j}^{\infty}-A_{i j}\left(\omega_{e}\right)\right] \omega_{e} \sin \left(\omega_{e} t\right) d \omega_{e} \\
\frac{2}{\pi} \int_{0}^{\infty}\left[B_{i j}\left(\omega_{e}\right)-B_{i j}^{\infty}\right] \cos \left(\omega_{e} t\right) d \omega_{e}
\end{array}\right.
$$

The diffraction modal force can be expressed in a similar way

$$
F_{i}^{d i f}=\int_{-\infty}^{\infty} \kappa_{i}^{D}(t-\tau) \zeta_{0}(\tau) d \tau,
$$

and

$$
\begin{aligned}
\kappa_{i}^{D}(t)=\frac{1}{2 \pi} \int_{-\infty}^{\infty} & {\left[\operatorname{Re}\left\{E_{i}^{D}\left(\omega_{e}\right)\right\} \cos \left(\omega_{e} t\right)\right.} \\
+ & \left.\operatorname{Im}\left\{E_{i}^{D}\left(\omega_{e}\right)\right\} \sin \left(\omega_{e} t\right)\right] d \omega_{e}
\end{aligned}
$$

Accuracy of the retardation function for radiation and diffraction modal force will directly affect the analysis results and development of reliable algorithm for the infinite integration is one of challenges in this time domain model.

In time domain hydroelastic BEM model, nonlinear rigid body motion Eqs. (9) and (10), are applied. The nonlinear terms in modal force computation need to be consistently involved as well.

\section{Examples of Application}

Two hydroelasticity codes have been developed in Lloyd's Register, HydroE-FD for the frequency domain model and HydroE-TD for the time domain model. The results of frequency domain hydroelastic analysis are compared against the results of Lloyd's Register's rigid body frequency domain code, WAVELOAD-FD in this section as well. The structure analysis was performed by using one of Lloyd's Register's FEA tools, Trident.

A container ship was selected as an analysis example. Particulars of this vessel, including ship dimensions, draft at fore and aft perpendicular, displacement, COG and radii of gyrations, are shown 
in Table 1.

The FEA mode and hydroelastic panel model are shown in Fig. 1. One meter size panel model was selected to capture the responses on high wave frequency range. Figs. 2 and 3 plot the shape of first 10 wettable elastic dry eigen modes and their $n$-term. The $n$-term of rigid motion mode roll and pitch are also presented in Fig. 3. It can be found that mode 9, 21 and 28 are three vertical bending modes, and all others are horizontal mode of bending, torque or their combinations.

The first result is the comparison of vessel deformation due to hydrostatic pressure and gravitational force. In Fig. 4, top plot came from the direct FEA analysis and bottom plot is the results from HydroE-FD. Static modal amplitude was presented in Table 2. The main contribution to the static deformation was from the three vertical bending modes as the model is very close to a symmetric case. The ship is in a static hogging state and maximum static deformation by direct FEA using Trident was $324 \mathrm{~mm}$ while that from HydroE-FD was $323 \mathrm{~mm}$, they are collocated well.

Natural frequency of each elastic eigen mode is one of most important results in a structural assessment, and the results of the "dry natural frequency" and "wave making natural frequency" are listed in Table 3. The "dry natural frequency" of those modes represents the natural frequency when the structure oscillates in air and they are the eigen values of the original FEA model. The "wave making natural frequency" is the natural frequency when the structure oscillates on free surface of water and generates the so-called radiation waves. The restoring force and encounter frequency depended wave making added-mass are considered together with the modal that the wave making effect always decreases natural frequency and it even changes the sequence of mass and stiffness.

From the results, we can see some eigen modes. The first vertical mode is the third elastic eigen mode in the original dry eigen mode list, but it jumps up to the second elastic mode in the wave making list due to the significant increase of mass by its wave making added-mass. Ocean wave is phenomenon of typical period in order of 10 seconds, and it in general does not directly excite resonant oscillation for those modes with wave making frequency higher than $5 \mathrm{rad} / \mathrm{sec}$.

The Rayleigh coefficient of structure damping used in this example was set to $2.3 \%$ for the first vertical bending mode, mode 9 , and $0 \%$ for the second and third vertical damping mode, mode 27 and 31; as for the horizontal mode, $1.3 \%$ was used for mode 7 ; $3.3 \%$ for mode 8 and $1.2 \%$ for mode 10 . Other modes

Table 1 Particulars of container ship model.

\begin{tabular}{|l|l|}
\hline Lbp $(m)$ & 325.0 \\
\hline B $(m)$ & 43.8 \\
\hline T_fp $(m)$ & 11.075 \\
\hline T_ap $(m)$ & 11.405 \\
\hline Displacement $\left(m^{3}\right)$ & 94,428 \\
\hline Wetted hull area $\left(m^{2}\right)$ & 14,910 \\
\hline LOG $(m)$ & 154.5 \\
\hline VOG $(m)$ & 15.342 \\
\hline Kxx $(m)$ & 16.088 \\
\hline Kyy $(m)$ & 78.235 \\
\hline $\operatorname{Kzz}(m)$ & 78.343 \\
\hline $\operatorname{Kxz}(m)$ & 6.112 \\
\hline
\end{tabular}
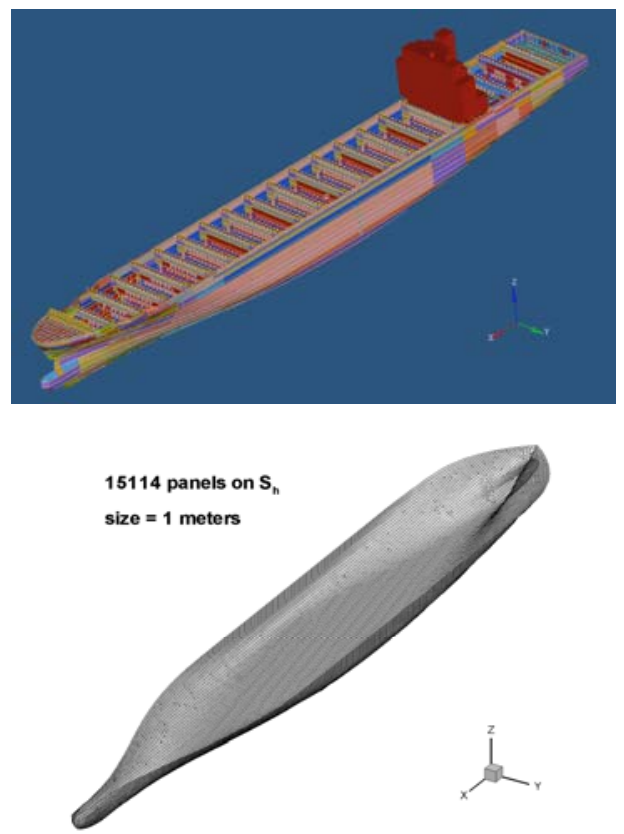

Fig. 1 FEA model (top) and hydroelastic panel model (bottom) of the container vessel. 

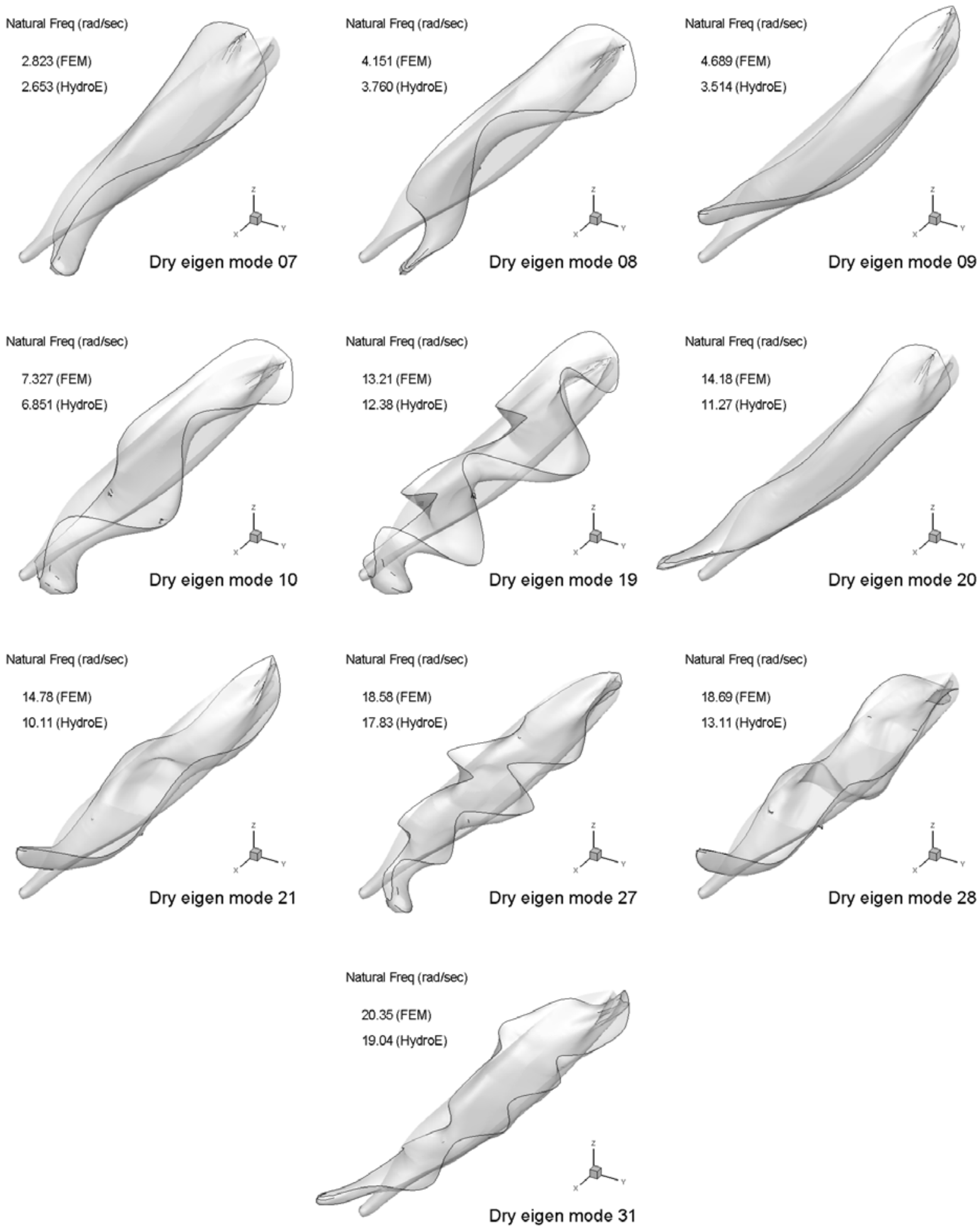

Fig. 2 Wetted hull surface and the natural frequency of the first $\mathbf{1 0}$ elastic modes of the container ship model. 

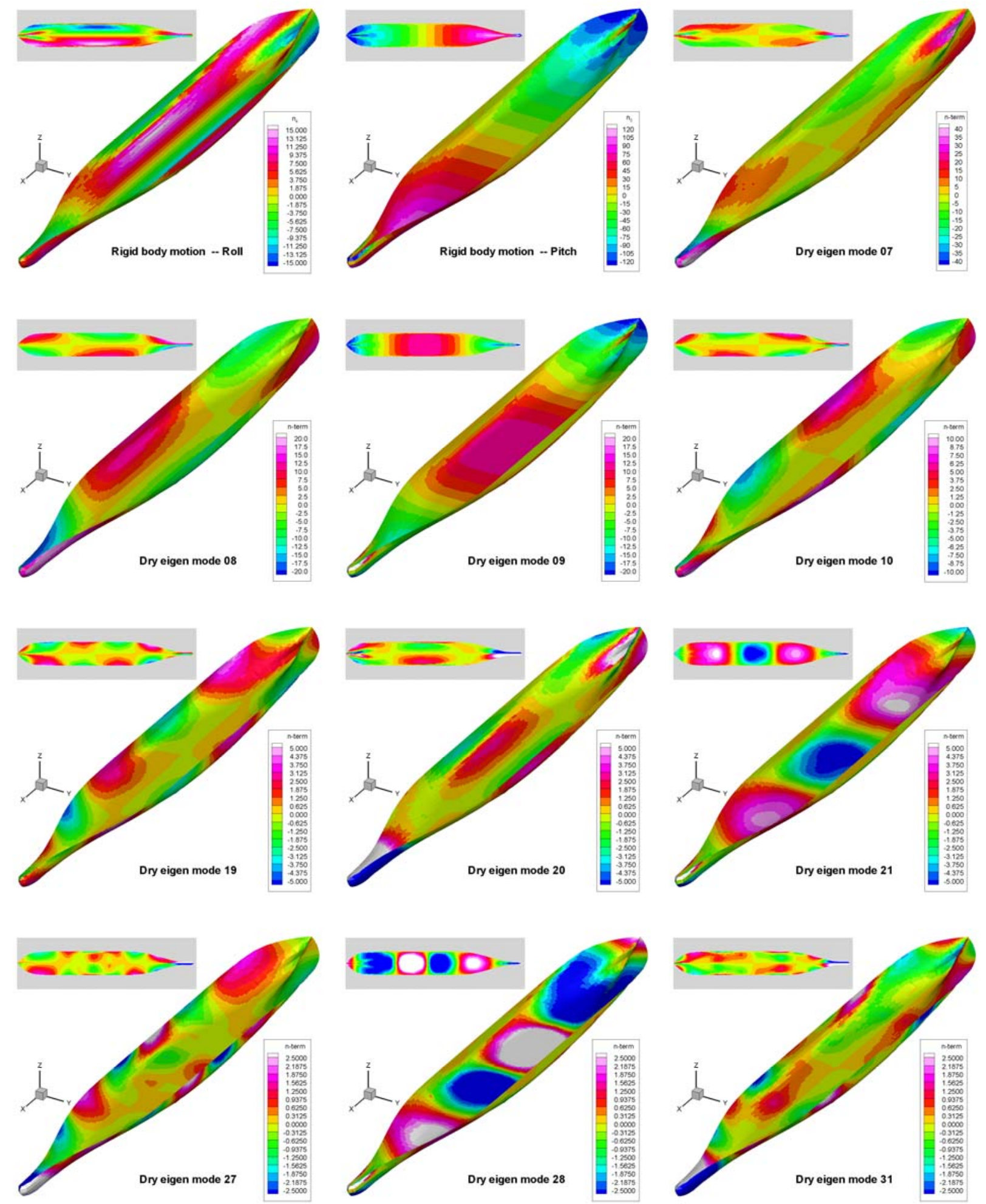

Fig. 3 The $n$-term of roll, pitch, and the first 10 elastic dry eigen modes. 


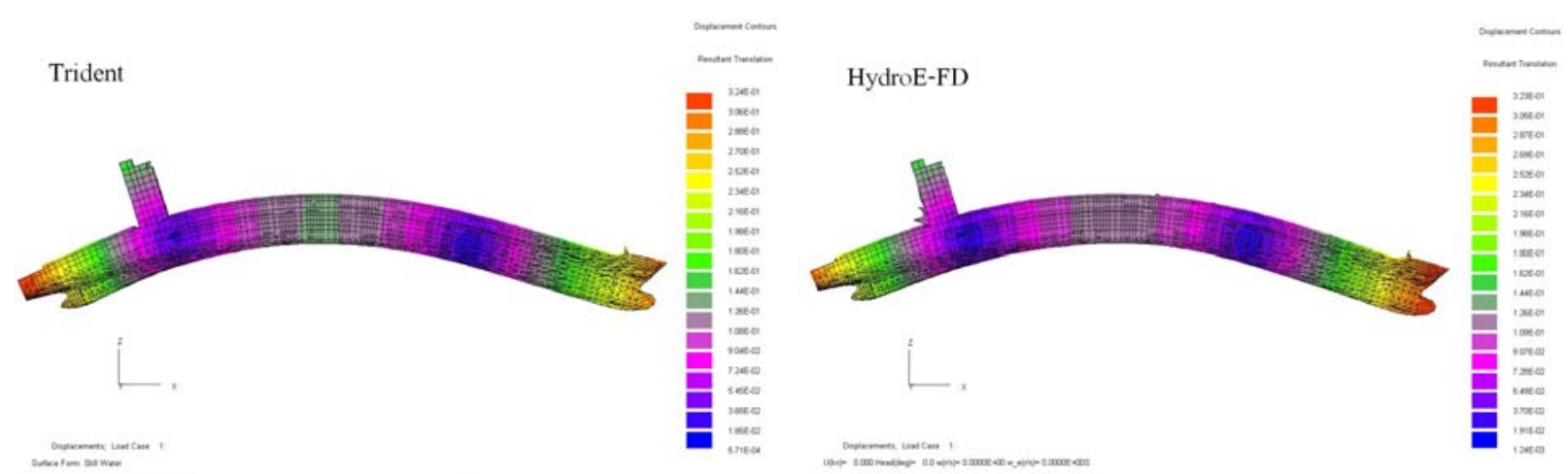

Fig. 4 Static displacement of the model by Trident (top) and HydroE-FD (bottom).

Table 2 Static mode amplitude by Hydro-FD.

\begin{tabular}{lllllllllll}
\hline Mode & 07 & 08 & 09 & 10 & 19 & 20 & 21 & 27 & 28 & 31 \\
\hline$\xi_{j}^{S}$ & 7.975 & 11.30 & -101.0 & 0.2505 & -0.1286 & 1.077 & -39.87 & -0.689 & 25.46 & -0.03120 \\
\hline
\end{tabular}

Table 2 Natural frequency of the container ship.

\begin{tabular}{lll}
\hline Eigen Mode & Dry natural frequency from FEA (rad/sec) & Wave making natural frequency from HydroE-FD (rad/sec) \\
\hline 07 & 2.823 & 2.655 \\
08 & 4.151 & 3.754 \\
09 & 4.689 & $\underline{3.501}$ \\
10 & 7.327 & 6.851 \\
19 & 13.21 & 12.38 \\
20 & 14.18 & $\underline{11.27}$ \\
21 & 14.78 & $\underline{10.11}$ \\
27 & 18.58 & 17.83 \\
28 & 18.69 & $\underline{13.11}$ \\
31 & 20.35 & 19.04 \\
\hline
\end{tabular}

horizontal modes had small effects on the global hydroelastic response.

RAO, the so-called Response Amplitude Operator which represents the response amplitude induced by wave of 1 meters amplitude, of rigid-body motion modes and elastic deformation modes over a range of incident wave frequency $[0.0,4.0] \mathrm{rad} / \mathrm{sec}$ and 150 degree of heading at three ship speeds are plotted in Fig. 5. Resonant responses of mode 7, 8 and 9 can be predicted in a range of low incident wave frequency [0, 1.6] rad/sec for the 20 knots case.

Distribution of hydrodynamic pressure RAO is shown in Fig. 6 in a resonant condition of the $3^{\text {rd }}$ elastic eigen mode, the first vertical bending mode in fact. Comparing to the rigid-body analysis results, hydroelastic analysis received much higher pressure in this case. Noting the n-term pattern shown in Fig. 3, we can find that pattern of the pressure distribution is similar to that of the n-term of the resonant eigen mode, and the radiation pressure is the dominant component in this case.

The modal resonant is determined by two factors, frequency and strength of external excitation. For the case of a ship, an elastic structural eigen mode can be excited when incident wave provides an environment to make the structure has an encounter frequency close to its modal natural frequency. The scale of this resonant eigen response, on the other hand, is determined strongly by the pattern of external exciting pressure, including pressure of incident wave and diffraction wave and the radiation wave of other mode as well. As found in Fig. 5, the third elastic eigen mode, the $1^{\text {st }}$ vertical bending mode in fact, has a resonant amplitude around 6.5 for the zero speed case 

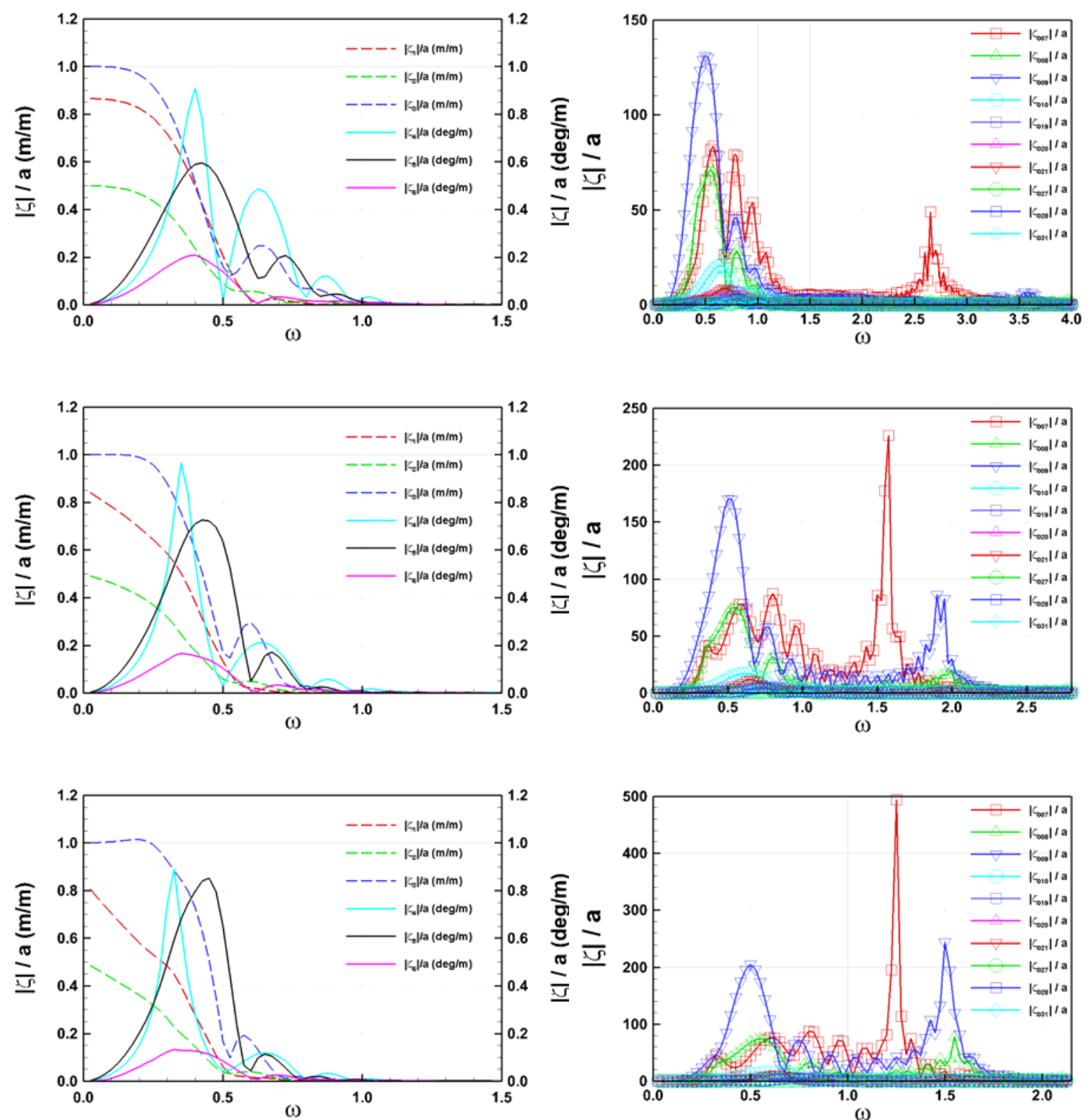

Fig. 5 RAO of rigid-body motion and elastic deformation in waves of $\chi=150^{\circ}$.

$1^{\text {st }}$ row: $U=0$ knots; $2^{\text {nd }}$ row: $U=10$ knots; $3^{\text {rd }}$ row: $U=20$ knots.

$1^{\text {st }}$ column: rigid body motion modes; $2^{\text {nd }}$ column: elastic deformation modes.

where the incident wave length is very short, around 5 meters. When the wave length increase to 28 meters in 20 knots case, amplitude of this eigen mode jumps up to a level of 50 time larger than that of zero ship speed.

Results of hydrodynamic pressure and acceleration of ship motion will directly change the results of internal loads. To check the effects of elastic deformation, internal load results of tension force, horizontal shearing force and vertical shearing force on a section at $25 \%$ of ship length from aft perpendicular are shown on the left column in Fig. 7; those of the torque, vertical bending moment and horizontal bending moment on mid-ship section are shown in the right column of the figure. Results of the frequency domain hydroelasticity model are in blue 

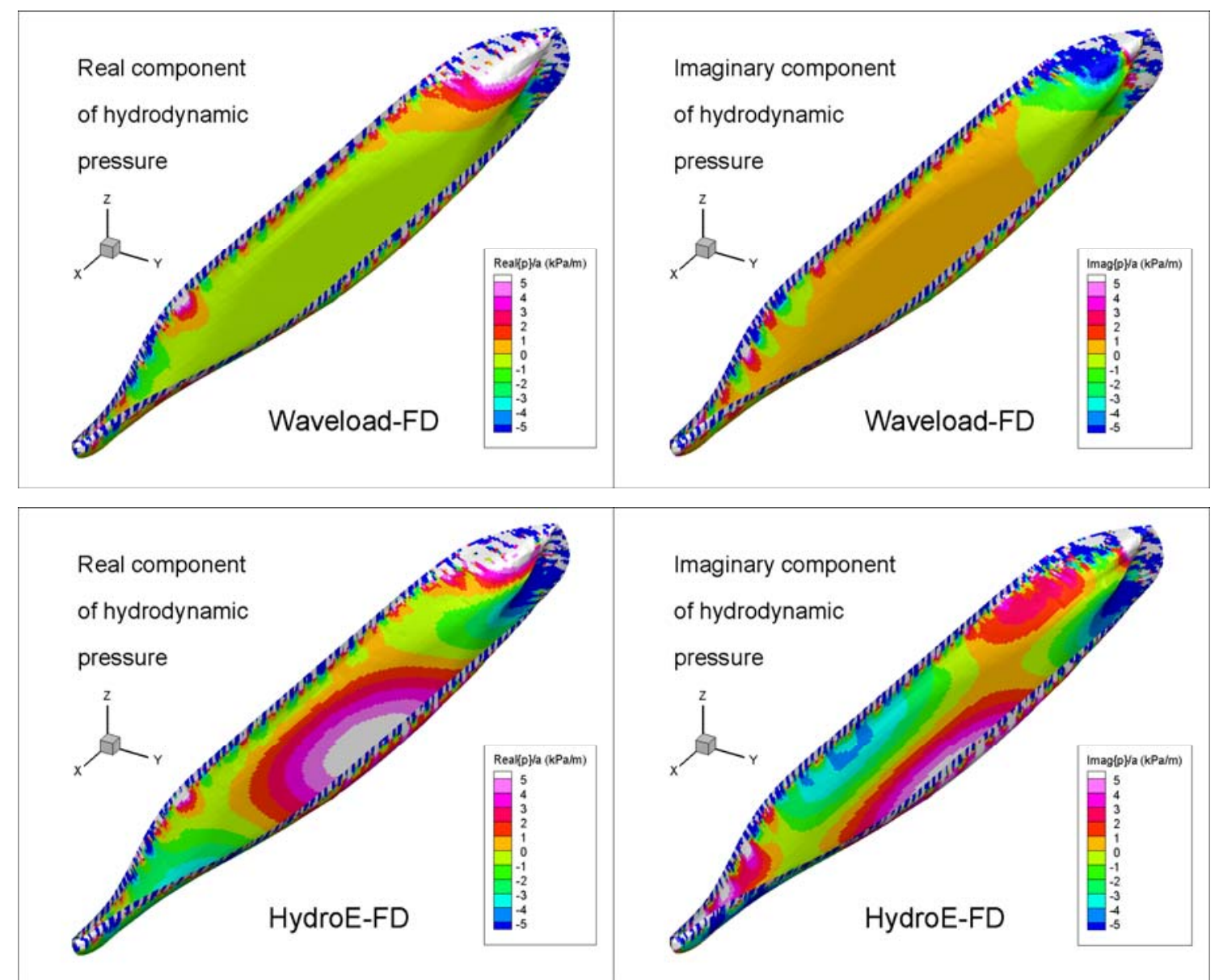

Fig. 6 RAO of hydrodynamic pressure of rigid body model (upper) and hydroelasticity model (lower) for condition $U=12 \mathrm{knots}, \chi=150^{\circ}$ and $\omega=1.875 \mathrm{rad} / \mathrm{s}$.

lines and the results from a rigid-body hydrodynamic analysis are shown in red diamond marks. From this figure, we can find that the internal loads by a hydroelasticity model will be close to the results by rigid-body analysis model when the ship is in an environment away from its structural nature frequencies, while when incident waves provide an environment of ship encounter frequency close to the structural nature frequency, the significant feedback can be expected from the internal loads, and those resonant internal loads will induce structure vibration, so-called springing, and make damage on the ship structure. For curtain types of ship or floating structure, fatigue life based on hydroelasticity assessment can be more than $30 \%$ shorter than that by a rigid-body based analysis. A hydroelastic assessment will definitely be required for such cases.
From results of frequency domain hydroelastic analysis, one can find the necessity of applying hydroelasticity and the impact of a hydroelasticity analysis in an engineering assessment. We should bear in mind that the frequency domain hydroelasticity is based on a "linear response" condition and works well for cases of water waves with small wave steepness. Time domain hydroelasticity model needs to be considered for large wave cases. We present some results of time domain hydroelasticity analysis for the same ship model in following. Time trace of four rigid-body motion modes is shown in Fig. 8, it is for a case in waves of 120 degree heading, $0.375 \mathrm{rad} / \mathrm{sec}$ of frequency and 20 meters wave height, and ship speed is 10 knots. We can see the time domain model received larger motion and nonlinear characteristics for pitch in this extremely high wave condition. The 


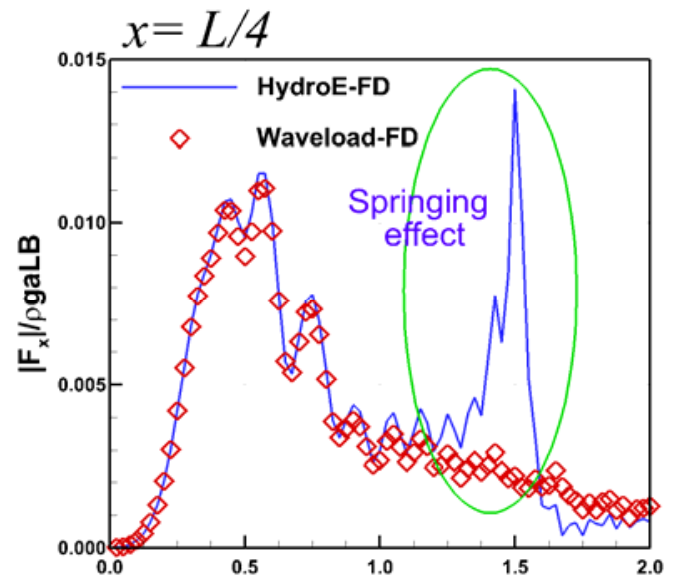

$\omega$
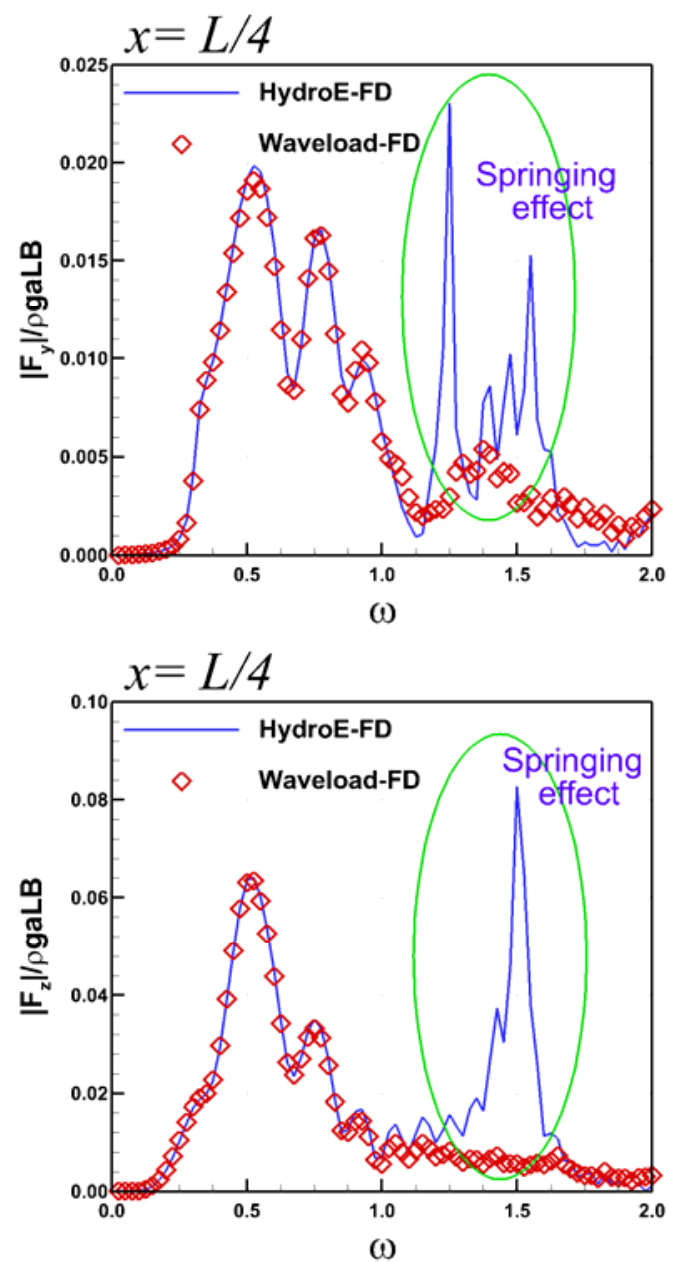

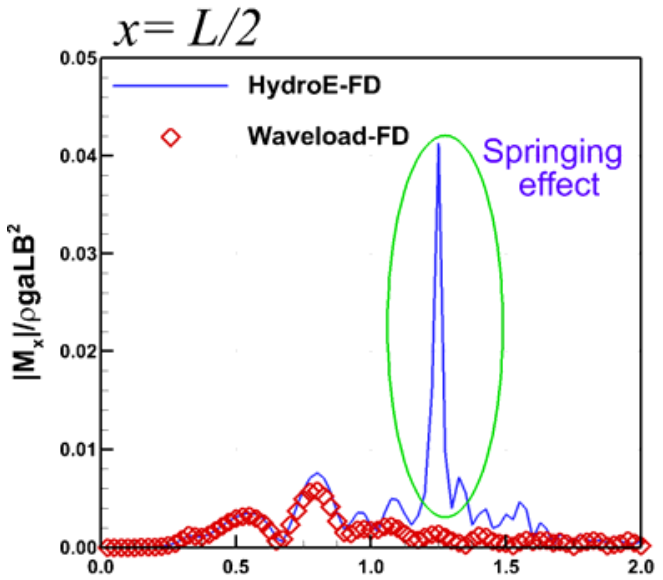

$\omega$

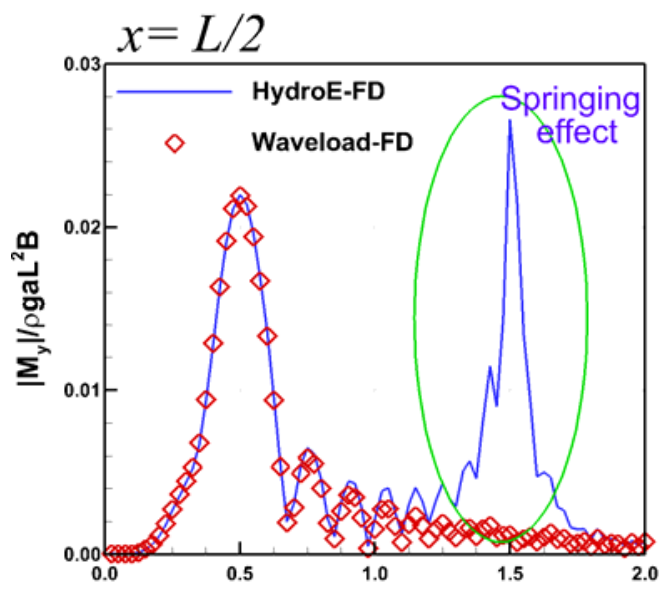

$\omega$

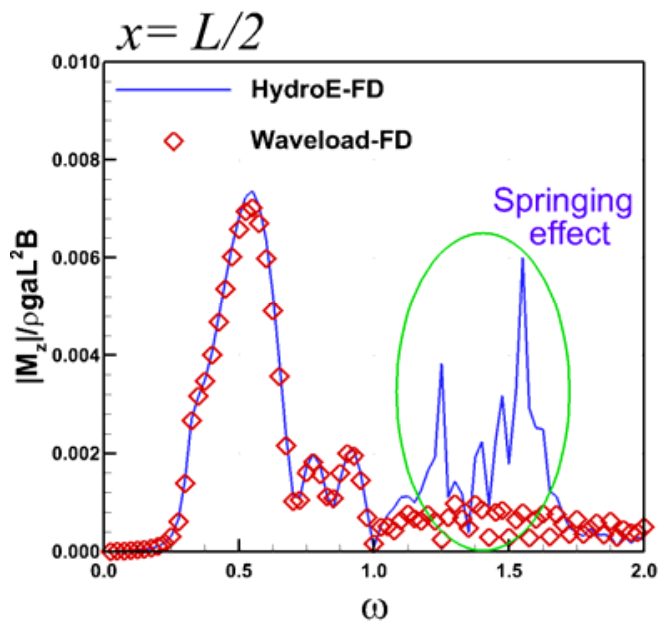

Fig. 7 Non-dimensional internal load in condition of $\mathbf{U}=20$ knots and $\chi=150^{\circ}$.

differences of the results from frequency domain analysis and time domain analysis are caused mainly by the so-called geometry nonlinear, i.e. the effect of "flare bow" and "flat stern" for present model.
Frequency domain model and time domain will have similar response prediction if the wettable surface of structure has purely vertical shape, but it is not the case for most of ships. Another reason causing this 


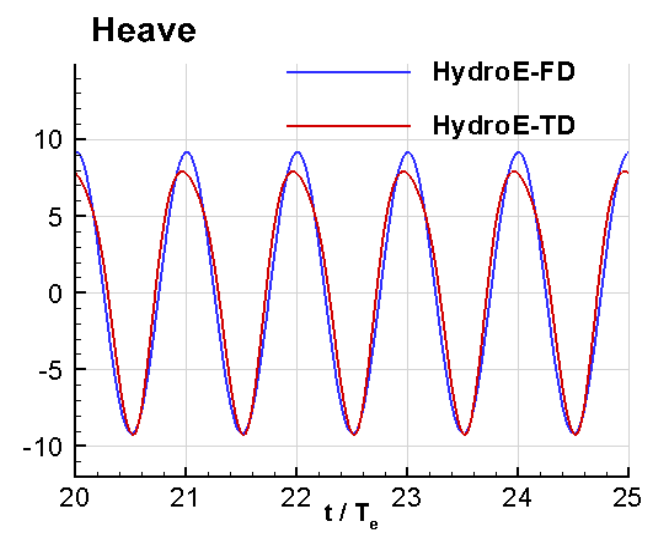

Pitch

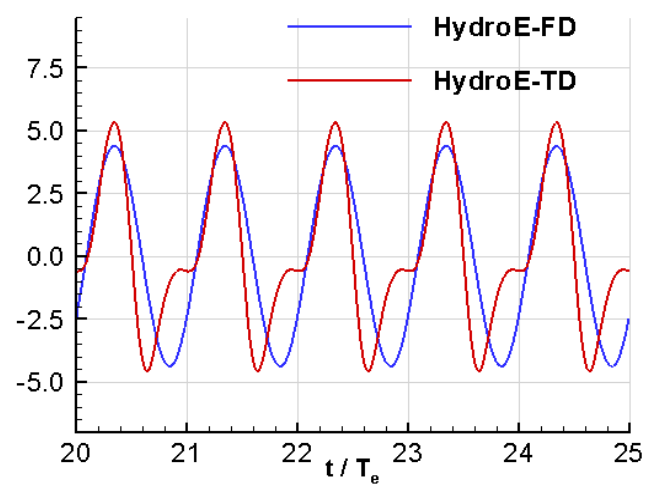

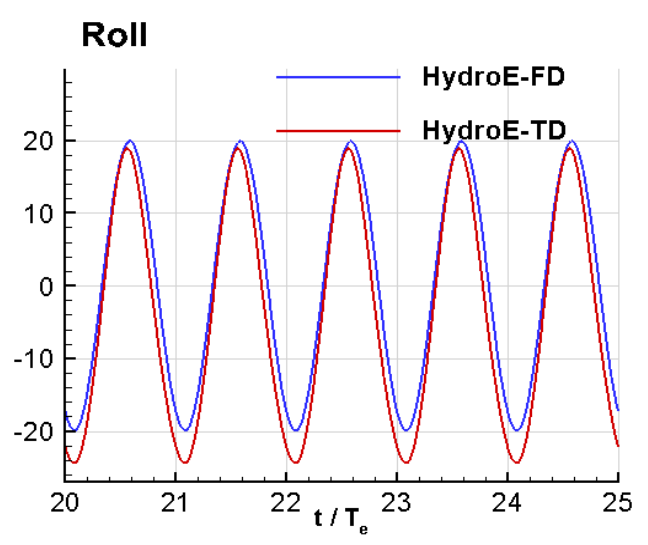

Yaw

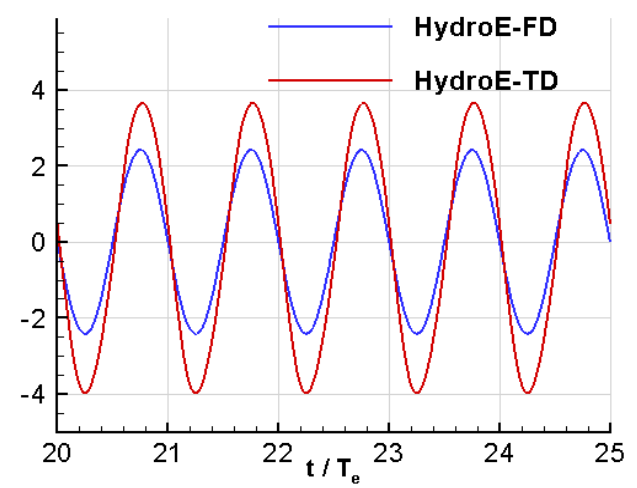

Fig. 8 Amplitude of some rigid-body motion modes in condition $\mathbf{U}=\mathbf{1 0} \mathrm{knots}, \chi=120^{\circ}, \omega=0.375 \mathrm{rad} / \mathrm{sec}$ and wave height $=20$ meters.

difference is the nonlinear large rotation motion. For example, the roll motion amplitude of present case goes up to 20 degrees, and it will affect the yaw motion. The effects of roll on yaw in the nonlinear rotation term $\mathbf{w} \times \mathbf{J}(\mathbf{w})$ and mapping matrix $\mathbf{R}$ are ignored in a frequency domain model therefore coupling between rotation motions are weaker there.

The most obvious differences of the two hydroelastic models are the pressure distribution and shown in Fig. 9. Hydrodynamic pressure is acting on the mean wetted surface of the ship in the frequency domain mode and changing in a sinusoidal style. From the top row of Fig. 9, we can see that there is no pressure on the mean dry hull surface, and the total pressure on bow can go have a negative value in a frequency domain model; on the other hand, pressure by a time domain model has no such problems and closer to the reality. A problem in frequency domain model is the "symmetric vertical load". Scale of dynamic vertical bending moment and shearing force will be the same for hogging and sagging condition from a frequency domain, and it is not the case in the real case especially when wave goes higher. For a conventional ship, the dynamic vertical load in a sagging condition is always larger in general than that in a hogging condition. To improve that in a time domain model, we are simulate the instantaneous wetted surface is solved and pressure on the surface and the vertical load become much closer to the reality. Dynamic and total vertical shearing force on a section at 1/4 of ship length from bow are presented in the top row of Fig. 10, those for vertical bending moment on a mid-ship section are presented on the bottom row of the figure. It shows that in a sagging state, the dynamic vertical bending moment in this wave condition is as large as twice of that in a hogging state. The vertical shearing force has similar tendency. It worth to remind that results of this extreme wave case 

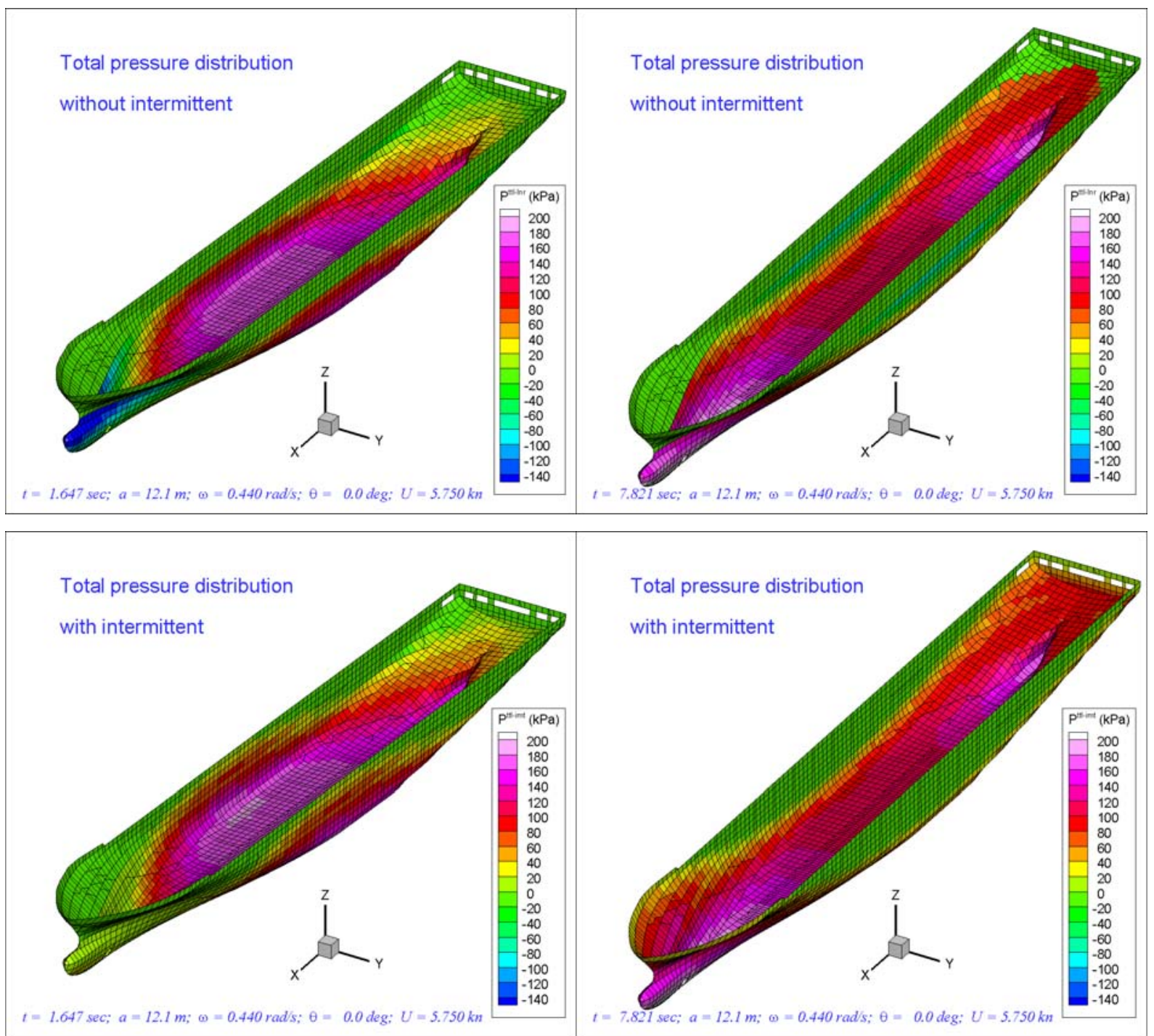

Fig. 9 Pressure distribution by frequency domain model (top) and time domain model (bottom) in condition $\mathrm{U}=5.75 \mathrm{knots}$, $\chi=0^{\circ}, \omega=0.44 \mathrm{rad} / \mathrm{sec}$ and wave height $=\mathbf{2 4 . 2}$ meters, in a hogging state (left) and a sagging state (right).
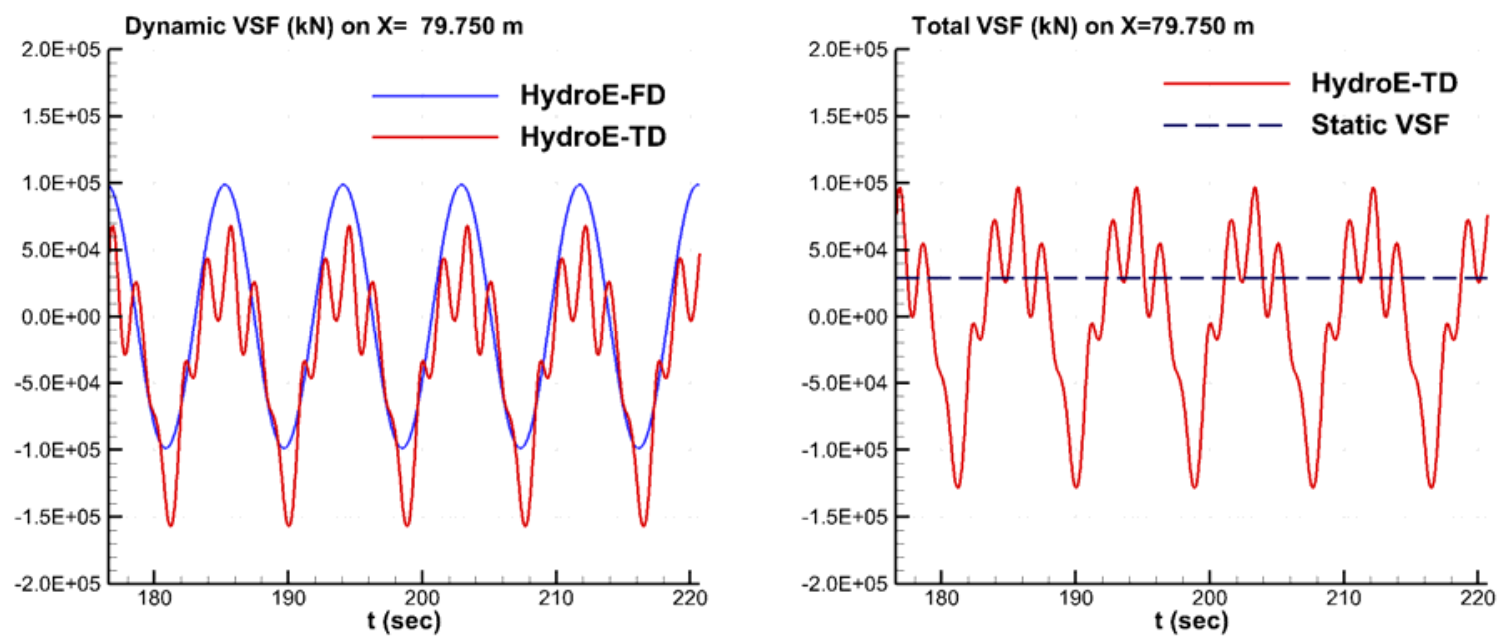

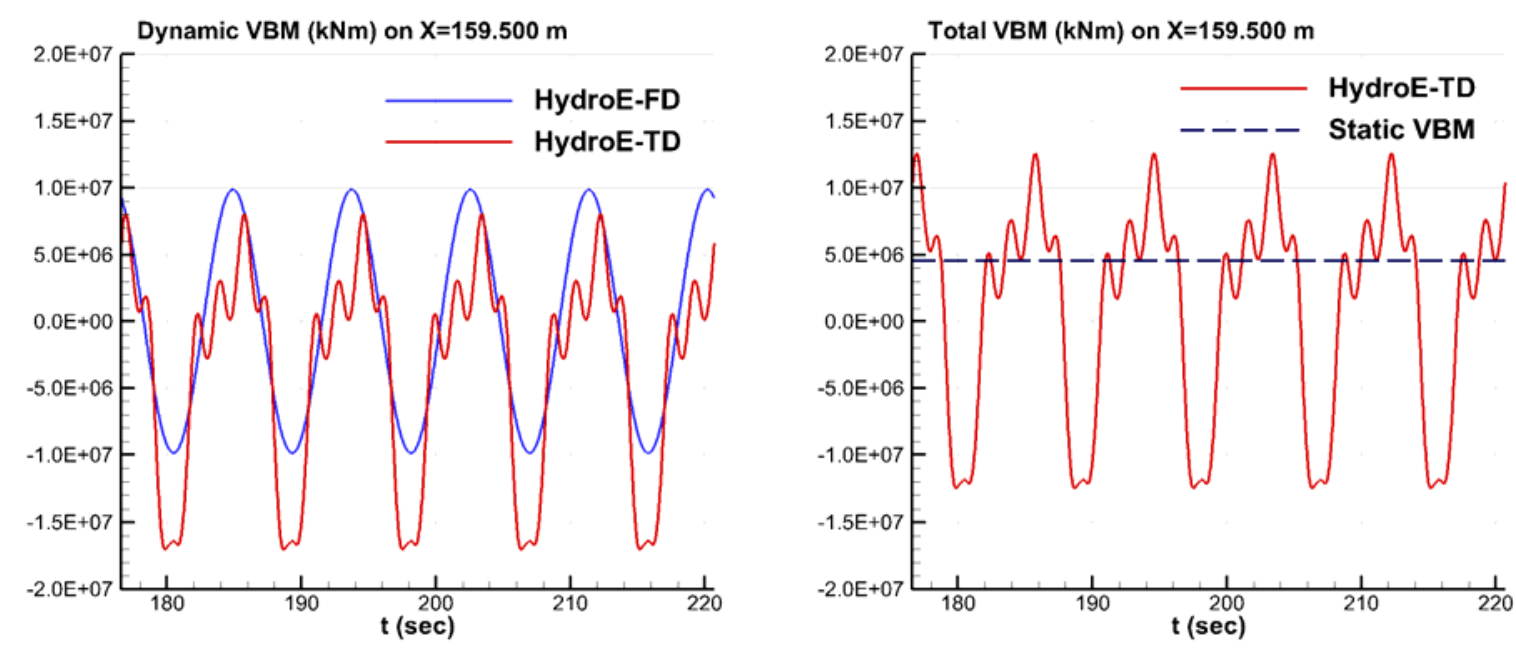

Fig. 10 Vertical shearing force and bending moment in condition $\mathbf{U}=20 \mathrm{knots}, \chi=180^{\circ}, \omega=0.475 \mathrm{rad} / \mathrm{sec}$ and wave height $=20$ meters.
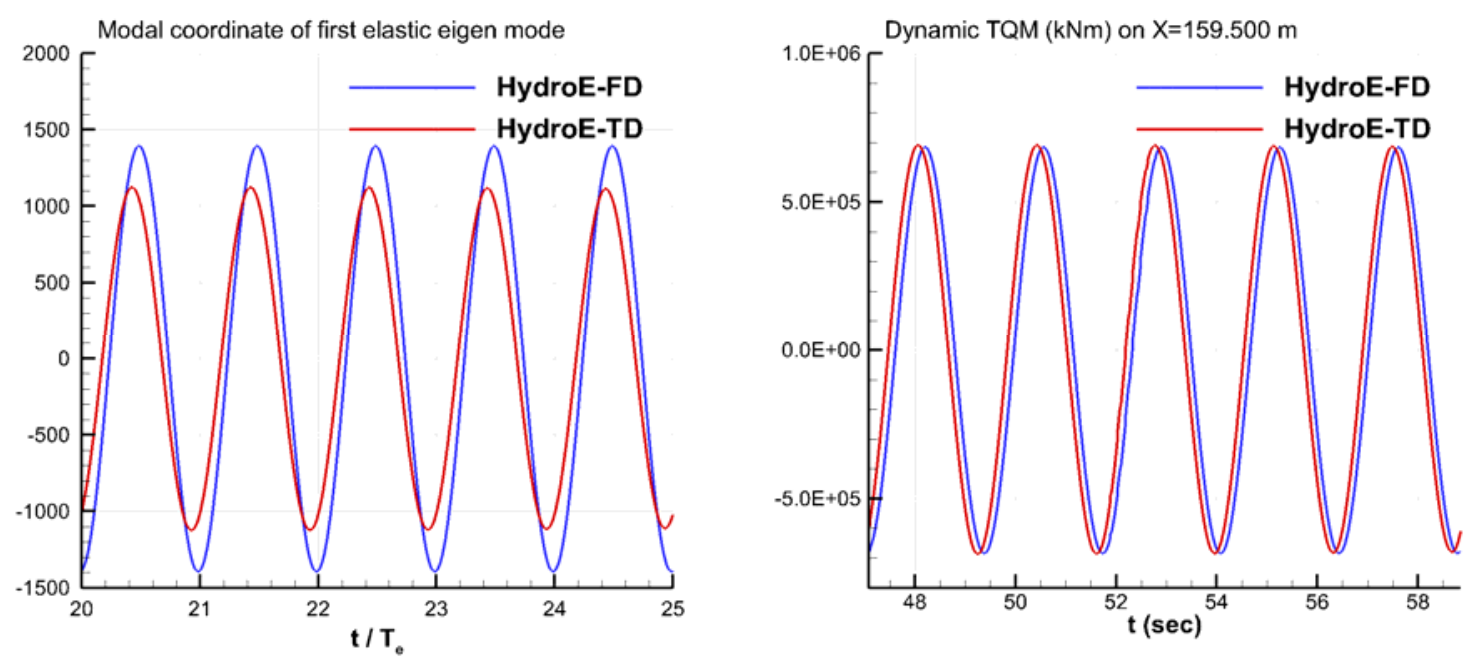

Fig. 11 Time trace of first elastic eigen mode amplitude and dynamic torque at mid-ship in condition $\mathrm{U}=20 \mathrm{knots,}$ $\chi=150^{\circ}, \omega=1.250 \mathrm{rad} / \mathrm{sec}$ (resonant frequency of the mode) and wave height $=2.82$ meter.

is for demonstration of the difference of frequency domain and time model, it is not a real case as there will be no ship operated at 20 knots in a 20 meters wave environment. The asymmetric ratio of vertical load in sagging/hogging condition will decrease when wave high become smaller.

The last result is the time trace of first elastic eigen mode, which is almost pure torque mode for present ship model, shown in the left of Fig. 11, and the time history of torque load on the mid-ship section. For this wave case, wave height is close to the breaking limitation, the amplitude of the first elastic mode by the time domain model is smaller than that by the frequency domain, but affected by the pressure correction in time domain approach, the torque load on the other hand are almost the same.

\section{Conclusions}

Theory of hydroelasticity is outlined in details for both linear frequency domain approach and non-linear time domain approach. Results of a container ship of 320 meters in length are used to demonstrate the improvement of hydrodynamic analysis from rigid-body to elastic body and from frequency domain to time 
domain. Those results also proved the importance to considering the application of hydroelastic analysis for assessment of ships or structures where structure vibration playing a dominant role.

The author would like to gratefully acknowledge the support of many colleagues in Lloyd's Register for their contributions to this work. The author also wishes to thank for the support from Global Technology Center of Lloyd's Register in Southampton and Applied Technology Group of Lloyd's Register to publish this work.

The views expressed in this work are those of the author alone and do not necessarily represent the policy of Lloyd's Register or any of its affiliates or subsidiaries.

\section{References}

[1] Bishop, R. E. D., and Price, W. G. 1979. Hydroelasticity of Ships. Cambridge: Cambridge University Press.

[2] Wu, Y. 1984. "Hydroelasticity of Floating Bodies." Ph.D. thesis, Brunel Univ., UK.

[3] Wang, D. 1996. "Three-Dimensional Hydroelastic Analysis of Ships in Time Domain.” Ph.D. thesis, China Ship Scientific Research Centre, China. 